\title{
Lattice gauge theory in technicolor
}

\author{
Benjamin Svetitsky \\ Raymond and Beverly Sackler School of Physics and Astronomy, Tel Aviv University, 69978 Tel Aviv, \\ Israel
}

\begin{abstract}
The methods of lattice gauge theory may be applied to gauge theories besides QCD, in fact to any gauge group and any representation of matter fields (as long as the coupling is not chiral). Such theories are useful for model building beyond the Standard Model, for instance in technicolor models. We have carried out Monte Carlo simulations of the SU(3) gauge theory with color sextet fermions. Our result for its discrete beta function indicate an infrared fixed point that makes the theory conformal rather than confining. The lattice theory's phase diagram shows no separation of chiral and confinement scales, measured when the quark mass is nonzero.
\end{abstract}

Key words: Lattice gauge theory, renormalization group, technicolor PACS: $11.15 . \mathrm{Ha}, 11.10 . \mathrm{Hi}, 12.60 . \mathrm{Nz}$

\section{Introduction}

The past year has seen an acceleration in the application of lattice gauge theory to models other than QCD, particularly to field theories that may find application in describing physics beyond the Standard Model [1. Gauge groups other than SU(3), and quark representations other than the fundamental, figure often in BSM models. We need to go beyond perturbation theory to verify whether a given model is suitable for technicolor, for instance, or whether it fails to confine quarks and thus becomes a theory of "unparticles." Supersymmetry also cries out for nonperturbative treatment. Current studies include $\mathrm{QCD}$ with various numbers of flavors, the $\mathrm{SU}(2)$ gauge theory with fermions in the adjoint representation, and the supersymmetric $\mathrm{SU}(2)$ gauge theory. I will describe here our study of the $\mathrm{SU}(3)$ gauge theory with color sextet quarks [2]3.

One should note significant limitations of lattice gauge theory or of its current implementations. For one thing, chiral gauge theories, so basic to weak interactions and beyond, have not yet found a lattice formulation (except for the Abelian theory [4]). Also, supersymmetric theories other than the simplest cannot be studied on a lattice without breaking supersymmetry severely. 


\section{SU(3) gauge theory with color sextet quarks}

We chose to study this model in order to settle two issues. The first is the question of the existence of an infrared fixed point (IRFP) that would render the infrared theory scale-invariant (rather than confining). The second is the possibility of scale separation, where the large Casimir operator of the sextet rep causes chiral symmetry to break spontaneously at an energy scale higher than the confinement scale. I will describe our work on the first question; for the second, suffice it to say that we have found no evidence for scale separation, but the issue isn't closed.

Caswell [5] noticed long ago that if you take QCD and increase the number of flavors $N_{f}$ beyond 8 then the two-loop term in the $\beta$ function is positive. This makes the $\beta$ function cross zero at a nonzero coupling $g_{*}$. If one takes the two-loop $\beta$ function to be the whole story, then this theory has an IRFP. The theory would be asymptotically free at high energies and scale-invariant at low energies, precluding confinement, chiral symmetry breakdown, a massive particle spectrum, and, indeed, any particles at all.

The same happens if you take quarks in the sextet rep with $N_{f}=2$ : The two-loop $\beta$ function indicates an IRFP at $g_{*}^{2} \simeq 10.4$. This is a rather strong coupling, which makes a two-loop result suspect. Nonetheless, the IRFP might survive in the full theory.

Or not: As one comes down in energy, the strengthening coupling might cause the chiral condensate to appear, whereupon the fermions would decouple and their contribution to the $\beta$ function would disappear. The theory would subsequently evolve as a pure gauge theory, consistent with confinement at large distances and the chiral condensate. This is what is needed for technicolor, and indeed this model has been proposed as a theory of "next-to-minimal" walking technicolor [6]

As it turns out, our numerical results argue for the first possibility, that the IRFP is real and there is no confinement.

\section{Schrödinger functional method}

Since the $\beta$ function is at the heart of the matter, we calculate it 2] (actually, a discrete version) on the lattice using the Schrödinger functional method [7, which is an implementation of the background field method. On a four-dimensional lattice of size $L^{4}$, we fix the gauge potential on the spacelike boundaries at times $t=0$ and $L$, which creates a classical background electric field throughout. We then calculate the free energy $\Gamma \equiv-\log Z$ (more precisely, a derivative of $\Gamma$ with respect to some parameter). Comparing the free energy to the classical action $S^{c l}$ of the background field then yields the running coupling $g^{2}(L)$ at the scale $L$, via $\Gamma=g(L)^{-2} S^{c l}$. Comparison of two lattices, with size $L$ and $2 L$, at the same lattice spacing $a$, gives the discrete beta function (DBF) for rescaling by a factor of two,

$$
B(u, 2)=\frac{K}{g^{2}(2 L)}-\frac{K}{g^{2}(L)},
$$

a function of $u \equiv K / g^{2}(L)$, where $K \simeq 37$ is connected to the classical action of our background field.

The result of comparing a lattice of size $4^{4}$ to one of size $8^{4}$ is shown in Fig. 1 The DBF crosses zero at $g^{2} \simeq 2.0$, a far cry from the two-loop estimate of $g^{2} \simeq 10$ and, all 


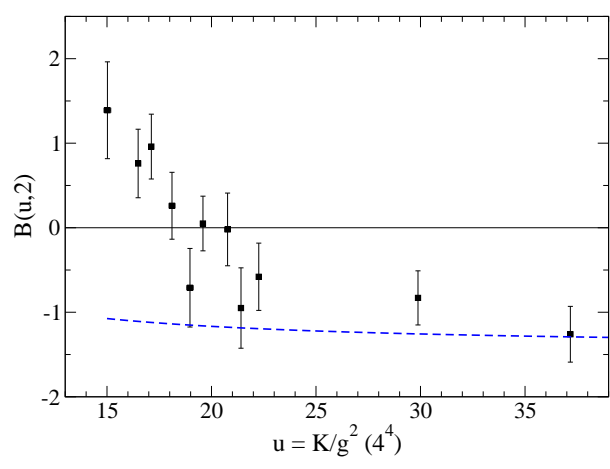

Fig. 1. Discrete beta function for $4 \rightarrow 8$.

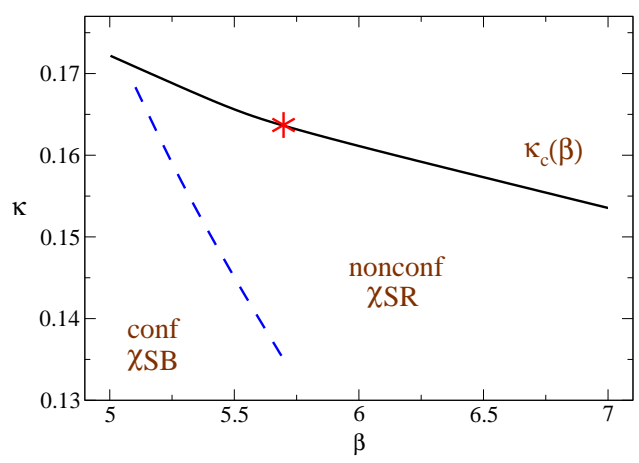

Fig. 2. Phase diagram with nonzero quark mass.

in all, a fairly weak coupling. If confirmed on larger lattices, this will indicate that the IRFP is a nonperturbative reality, and the theory is conformal (i.e., scale invariant) at large distances - not a candidate for technicolor.

\section{Caveat}

Lattice studies always have to be pushed to larger lattices. Here this is equivalent (for given physical lattice size $L$ ) to ever smaller lattice spacing. A lattice with $4^{4}$ sites is rather small; one might find reassurance in the fact that we find the same result in comparing $6^{4}$ with $8^{4}$. We are working on the DBF for $6 \rightarrow 12$, which, together with the $4 \rightarrow 8$ result shown, will furnish the beginning of a scaling study for scale ratio 2 .

Some more insight is offered [3] by a look at the theory with nonzero quark mass $m_{q}$. Here we can look for finite-temperature phase transitions to see what the physical scales are (see Fig. 2). We find that (1) there is only one phase transition (dashed line) for any lattice size studied, meaning no scale separation; and (2) our IRFP (the star on the $\kappa_{c}$ line, along which $m_{q}=0$ ) is always in the weak-coupling phase, indicating that a singlecoupling $\beta$ function may indeed give a valid description of the physics. The properties of the conformal theory at the IRFP offer fascinating questions for future work.

\section{References}

[1] See the proceedings of Lattice 2008, and in particular G. T. Fleming, PoS LATTICE2008, 021 (2008) arXiv:0812.2035 [hep-lat]].

[2] Y. Shamir, B. Svetitsky, and T. DeGrand, Phys. Rev. D 78, 031502 (2008) arXiv:0803.1707 [heplat]]; arXiv:0809.2885 [hep-lat].

[3] T. DeGrand, Y. Shamir, and B. Svetitsky, arXiv:0809.2953 [hep-lat]; arXiv:0812.1427 [hep-lat].

[4] M. Luscher, arXiv:hep-th/0102028.

[5] W. E. Caswell, Phys. Rev. Lett. 33, 244 (1974); T. Banks and A. Zaks, Nucl. Phys. B 196, 189 (1982).

[6] A. Belyaev, R. Foadi, M. T. Frandsen, M. Järvinen, A. Pukhov, and F. Sannino, arXiv:0809.0793 [hep-ph].

[7] R. Sommer, arXiv:hep-ph/9711243 